\title{
Dynamics and equilibration of Rydberg excitations in dissipative atomic ensembles
}

\author{
David Petrosyan \\ Institute of Electronic Structure and Laser, FORTH, GR-71110 Heraklion, Crete, Greece
}

(Dated: July 17, 2018)

\begin{abstract}
We study resonant optical excitations of strongly-interacting Rydberg states of atoms in the presence of relaxations. We employ the quantum stochastic (Monte Carlo) wavefunctions to simulate the dissipative dynamics of tens of atoms in two-dimensional lattices. We show that under typical experimental conditions involving slow Rydberg state decay and sizable relaxation of atomic coherences, on the time scale of several $\mu$ s the atomic ensemble approaches a stationary state in which much of the quantum correlations between the atoms have decayed away. The steady state, however, exhibits strong classical correlations of Rydberg excitation probabilities.
\end{abstract}

PACS numbers: $32.80 . \mathrm{Ee}, 32.80 . \mathrm{Rm}, 37.10 . \mathrm{Jk}$

Atoms in high-lying Rydberg states interact with each other via a long-range van der Waals (vdW) potential [1[3] which is many orders of magnitude stronger than the interaction potential between ground-state atoms at $\mu \mathrm{m}$ distances. In dense atomic ensembles $\left(\rho_{\text {at }} \gtrsim 10^{12} \mathrm{~cm}^{-3}\right)$, resonant optical excitations of multiple Rydberg atoms are then strongly suppressed [4 8] due to the interaction induced level shifts. In a small volume, where the interatomic interaction energies exceed the excitation linewidth of the Rydberg state, a single Rydberg atom blocks the excitation of all the other atoms 9 -14]. Larger atomic ensembles can accommodate more Rydberg excitations whose number exhibits reduced fluctuations [15, 16]. Recent experiments have spectacularly demonstrated Rabi oscillations of single Rydberg excitations in blockaded ensembles of atoms [13], and spatial ordering of multiple Rydberg excitations in larger ensembles of atoms in two-dimensional (2D) optical lattices [17.

In most experiments [6 $8,12,13,15-20]$, the Rydberg state is resonantly coupled to the atomic ground state by a two-photon transition via a non-resonant intermediate state. With the pulsed laser excitation, coherent dynamics of the many-atom system is observed on the $\mu$ s time scale [13, 17]. For comparison, typical lifetime of the highly excited Rydberg state with the principal quantum number $n \gtrsim 40$ is $\tau_{r} \sim 20 \mu \mathrm{s}$, while the intermediate state decay, two-photon laser linewidth, stray electromagnetic fields, atomic motion and collisions dephase the atomic polarization resulting in the coherence time of $\tau_{z} \lesssim 10 \mu \mathrm{s}$. It is therefore important to quantify the role of relaxations in the dynamical evolution of the system and its equilibration, and to characterize the properties of the resulting stationary state which may indeed be different from the transient or time-averaged state of a fully coherent (unitary) system.

Exact treatment of strongly-interacting many-body systems is computationally demanding - the dimension of Hilbert space for $N$ two-level atoms is $2^{N}$ —and even more so for dissipative systems. It requires the solution of $2^{2 N}$ density matrix equations which in practice is restricted to $N \lesssim 12$ atoms due to computer memory limitations. An alternative but equivalent approach employs the quantum stochastic (Monte Carlo) wavefunctions, which amounts to propagating $2^{N}$ amplitude equations, interrupted by quantum jumps, and then averaging over many independent trajectories. The quantum Monte Carlo simulations can thus deal with about double the number of atoms $N$ but require longer simulation times (or parallel computation) to generate many trajectories for good averaging. Furthermore, limiting the maximal number of excitations, which is justified by the Rydberg blockade, can greatly reduce the required Hilbert space dimension permitting an approximate treatment of many more atoms [16, 17, 21].

This paper presents the results of quantum Mote Carlo simulations for Rydberg excitation of several tens of atoms in 2D lattices. Typical experimental values 17 for the Rabi frequency of the driving field and the relaxation rates of atomic population and coherence are used in the simulations and the resulting dynamics is compared to that of a unitary (dissipationless) system. When all the atoms are within the blockade volume, the system undergoes damped Rabi oscillations between the collective ground and single Rydberg excitation states, and the final stationary state of the system contains a single Rydberg excitation with close to unit probability 22]. But when the system size is larger than the blockade volume, the weaker interactions between distant atoms cannot block multiple Rydberg excitations; instead they dephase the Rabi oscillations even in the unitary system. Including relaxations with rates comparable to the single-atom Rabi frequency steers the system towards a stationary state in which the quantum correlations between the atoms have largely decayed away. Strong classical correlations, however, persist, as the steady state is characterized by very small fluctuations of the number of excitations which is consistent with the tight spatial ordering of Rydberg excitations [17, 22].

Let us now turn to the quantitative description of the system. A 2D lattice of $N$ atoms is irradiated by a uniform laser field which resonantly drives the transition from the atomic ground state $|g\rangle$ to the highly excited Rydberg state $|r\rangle$ with Rabi frequency $\Omega$. The atom-field 
interaction Hamiltonian reads

$$
\mathcal{V}_{\mathrm{af}}^{j}=-\hbar \Omega\left(\hat{\sigma}_{r g}^{j}+\hat{\sigma}_{g r}^{j}\right),
$$

where $\hat{\sigma}_{\mu \nu}^{j} \equiv|\mu\rangle_{j j}\langle\nu|$ is the transition operator for atom $j$. The relaxation processes affecting each atom include the population decay of the excited state $|r\rangle$ with rate $\Gamma_{r}$, and the decay of atomic coherence $\hat{\sigma}_{r g}$ with rate $\Gamma_{z}$. These processes are described by Liouvillians in the Lindblad form

$$
\mathcal{L}^{j} \hat{\rho}=\frac{1}{2}\left[2 \hat{L}^{j} \hat{\rho} \hat{L}^{j \dagger}-\left\{\hat{L}^{j \dagger} \hat{L}^{j}, \hat{\rho}\right\}\right],
$$

where $\hat{\rho}$ is the density operator of the system, while the generators for the population and coherence decay are given, respectively, by $\hat{L}_{r}^{j}=\sqrt{\Gamma_{r}} \hat{\sigma}_{g r}^{j}$ and $\hat{L}_{z}^{j}=\sqrt{\Gamma_{z}}\left(\hat{\sigma}_{r r}^{j}-\right.$ $\hat{\sigma}_{g g}^{j}$ ). For an isolated atom, the (steady-state) excitation linewidth of state $|r\rangle$ is $w \simeq 2 \Omega \sqrt{\gamma_{r g} / \Gamma_{r}}$ with $\gamma_{r g} \equiv$ $\frac{1}{2} \Gamma_{r}+2 \Gamma_{z}$ and assuming $\Omega^{2}>\Gamma_{r} \gamma_{r g}[22,23]$.

We next include the interatomic interactions. The $\mathrm{vdW}$ potential between a pair of atoms $i$ and $j$ at positions $\mathbf{x}_{i}$ and $\mathbf{x}_{j}$ induces the level shift $\Delta\left(\mathbf{x}_{i}-\mathbf{x}_{j}\right)=$ $C_{6}\left|\mathbf{x}_{i}-\mathbf{x}_{j}\right|^{-6}$ of state $\left|r_{i} r_{j}\right\rangle$, where $C_{6}$ is the vdW coefficient 24]. The corresponding atom-atom interaction Hamiltonian reads

$$
\mathcal{V}_{\mathrm{aa}}^{i j}=\hbar \hat{\sigma}_{r r}^{i} \Delta\left(\mathbf{x}_{i}-\mathbf{x}_{j}\right) \hat{\sigma}_{r r}^{j} .
$$

When the vdW level shift is larger than the Rydberg state excitation linewidth, $\Delta \gtrsim w$, an atom in state $|r\rangle$ blocks the excitation of another atom [2, 3, 9]. We can then define the blockade distance $d_{\mathrm{b}}$ via $\Delta\left(d_{\mathrm{b}}\right)=w$, which yields $d_{\mathrm{b}} \equiv \sqrt[6]{C_{p} / w}$.

The total Hamiltonian for $N$ atoms is given by

$$
\mathcal{H}=\sum_{j}^{N} \mathcal{V}_{\text {af }}^{j}+\sum_{i<j}^{N} \mathcal{V}_{\text {aa }}^{i j} .
$$

To simulate the dissipative dynamics of the many-body system, we employ the quantum Monte Carlo wavefunctions [23, 25]. In such simulation, the state of the system $|\Psi\rangle$ evolves according to the Schrödinger equation $\partial_{t}|\Psi\rangle=-\frac{i}{\hbar} \tilde{\mathcal{H}}|\Psi\rangle$ with an effective Hamiltonian

$$
\tilde{\mathcal{H}}=\mathcal{H}-\frac{i}{2} \hbar \hat{L}^{2}
$$

where

$$
\hat{L}^{2} \equiv \sum_{j}\left(\hat{L}_{r}^{j \dagger} \hat{L}_{r}^{j}+\hat{L}_{z}^{j \dagger} \hat{L}_{z}^{j}\right)=\sum_{j} \Gamma_{r} \hat{\sigma}_{r r}^{j}+\Gamma_{z} \mathbb{1}
$$

is the non-Hermitian part which does not preserve the norm of the wavefunction $|\Psi\rangle$ during the evolution. The evolution is interrupted by random quantum jumps $|\Psi\rangle \rightarrow \hat{L}_{r, z}^{j}|\Psi\rangle / P_{r, z}^{j}$ with probabilities determined by the corresponding weights $P_{r, z}^{j} \equiv\left\langle\Psi\left|\hat{L}_{r, z}^{j \dagger} \hat{L}_{r, z}^{j}\right| \Psi\right\rangle$. In a single quantum trajectory, the normalized wavefunction of the system at any time $t$ is given by
$|\bar{\Psi}(t)\rangle=|\Psi(t)\rangle / \sqrt{\langle\Psi(t) \mid \Psi(t)\rangle}$. The approximate density operator of the system is then obtained by averaging over many $M$ independently simulated trajectories, $\hat{\rho}(t)=\frac{1}{M} \sum_{m}^{M}\left|\bar{\Psi}_{m}(t)\right\rangle\left\langle\bar{\Psi}_{m}(t)\right|$. On the other hand, the steady-state density operator can be approximated as a long-time average over a single trajectory, $\hat{\rho}(\infty)=\lim _{t \rightarrow \infty} \frac{1}{t-t_{0}} \int_{t_{0}}^{t} d t|\bar{\Psi}(t)\rangle\langle\bar{\Psi}(t)|$.

The mean number of Rydberg excitations within an ensemble of $N$ atoms is $\left\langle n_{\mathrm{R}}\right\rangle=\left\langle\sum_{j}^{N} \hat{\sigma}_{r r}^{j}\right\rangle$, while the probabilities $p_{\mathrm{R}}(n)=\left\langle\hat{\Sigma}_{r}^{(n)}\right\rangle$ of $n$ excitations are defined through the corresponding projectors $\hat{\Sigma}_{r}^{(0)} \equiv \prod_{j}^{N} \hat{\sigma}_{g g}^{j}$, $\hat{\Sigma}_{r}^{(1)} \equiv \sum_{j}^{N} \hat{\sigma}_{r r}^{j} \prod_{i \neq j}^{N} \hat{\sigma}_{g g}^{j}$, etc. Obviously $\left\langle n_{\mathrm{R}}\right\rangle=$ $\sum_{n} n p_{\mathrm{R}}(n)$, while $\hat{\sigma}_{g g}^{i}+\hat{\sigma}_{r r}^{i}=\mathbb{1} \forall i \in[1, N]$. To quantify the probability distribution of Rydberg excitations, we use the Mandel $Q$ parameter [26]

$$
Q \equiv \frac{\left\langle n_{\mathrm{R}}^{2}\right\rangle-\left\langle n_{\mathrm{R}}\right\rangle^{2}}{\left\langle n_{\mathrm{R}}\right\rangle}-1,
$$

where $\left\langle n_{\mathrm{R}}^{2}\right\rangle=\sum_{n} n^{2} p_{\mathrm{R}}(n)$. A Poissonian distribution $p_{\mathrm{R}}(n)=\left\langle n_{\mathrm{R}}\right\rangle^{n} e^{-\left\langle n_{\mathrm{R}}\right\rangle} / n$ ! leads to $Q=0$, while $Q<0$ corresponds to sub-Poissonian distribution, with $Q=-1$ attained for a definite number $n$ of excitations, $p_{\mathrm{R}}(n)=$ 1.

We have performed numerical simulations for various number of atoms $N$ arranged in 2D circular volumes (disks) of different diameter $d \leq 2 d_{\mathrm{b}}$. In the dynamical simulations, we typically generate $M \sim 200$ trajectories for smooth averaging. We truncate the Hilbert space by limiting the maximal number of Rydberg excitations, but verify the convergence by including more excitations, at the expense of less accurate averaging (fewer trajectories and shorter evolution times). We use the parameters similar to those in the experiment [17], assuming cold ${ }^{87} \mathrm{Rb}$ atoms with the ground state $|g\rangle \equiv 5 S_{1 / 2} \mid F=2, m_{F}=$ $-2\rangle$ and the Rydberg state $|r\rangle \equiv n S_{1 / 2}$ with the principal quantum number $n$. The resonant excitation of $|r\rangle$ is effected by a two-photon transition via non-resonant intermediate state $|e\rangle=5 P_{3 / 2}\left|F=3, m_{F}=-3\right\rangle$ with Rabi frequency $\Omega=2 \pi \times 85 \mathrm{kHz}$ (our definition of the Rabi frequency in $\mathcal{V}_{\text {af }}^{j}$ differs from that in [17] by a factor of $\frac{1}{2}$ ). The (population) decay rate of the Rydberg state $|r\rangle$ is $\Gamma_{r} \simeq 0.075 \Omega=40 \mathrm{kHz}$ [17]. The coherence relaxation $\Gamma_{z} \simeq 0.3 \Omega=160 \mathrm{kHz}$ on the transition $|g\rangle \leftrightarrow|r\rangle$ has two main contributions: the decay from the intermediate state $|e\rangle \rightarrow|g\rangle$ with the rate $\sim 90 \mathrm{kHz}$ and the two-photon laser linewidth $\sim 70 \mathrm{kHz}$, while at $T \sim 10 \mathrm{nK}$ 17] the Doppler broadening is negligible $(\lesssim 10 \mathrm{kHz})$. The resulting (steady-state) excitation linewidth of $|r\rangle$ is $w \simeq 2 \pi \times 0.5 \mathrm{MHz}\left(w \simeq 2 \pi \times 120 \mathrm{kHz}\right.$ if $\left.\Gamma_{z}=0\right)$. The corresponding blockade distance for the Rydberg states with $n \simeq 40-50\left(C_{6} / 2 \pi \simeq 1-15 \mathrm{GHz} \mu \mathrm{m}^{6}\right.$ [24] $)$ is $d_{\mathrm{b}} \sim 3.5-5.6 \mu \mathrm{m}$.

Consider first the case of a small confinement volume, $d<d_{\mathrm{b}}$. Due to the blockade effect, such a volume can accommodate at most one Rydberg excitation [9, 13]. Figure 1 shows the dynamics of the system and the steady 


\section{(a)}
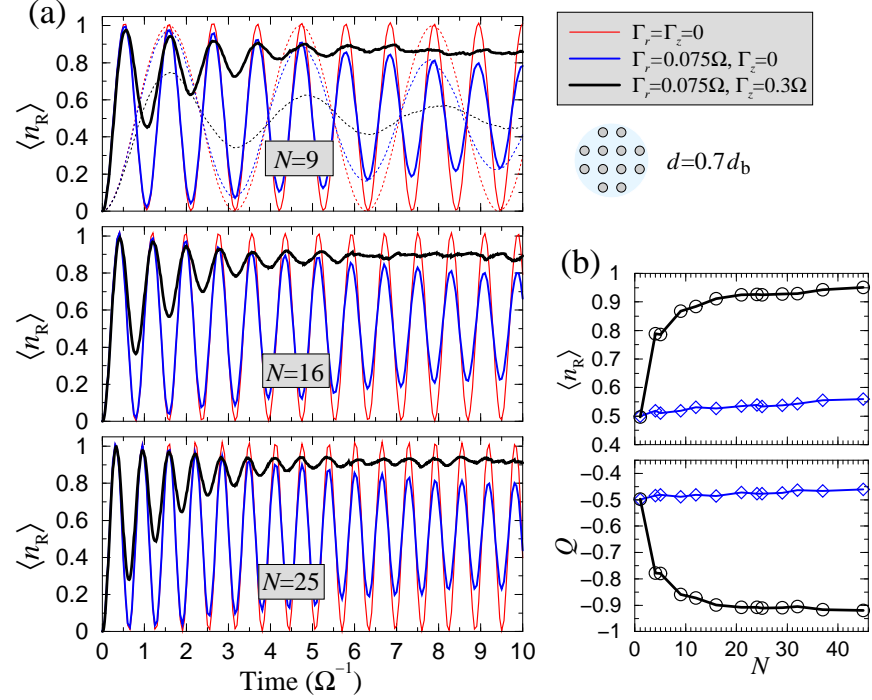

FIG. 1. Dynamics and steady-state of resonantly-driven ensemble of $N$ atoms in a $2 \mathrm{D}$ lattice of diameter $d=0.7 d_{\mathrm{b}}$. (a) Time-dependence of the number of Rydberg excitations $\left\langle n_{\mathrm{R}}\right\rangle$ for $N=9,16,25$ and different $\Gamma_{r, z}$. Thin dotted lines in the top graph correspond to a single atom, $N=1$. Time is in units of $\Omega^{-1} \simeq 1.87 \mu \mathrm{s}$. (b) Steady state values of $\left\langle n_{\mathrm{R}}\right\rangle$ and $Q$ versus $N \leq 45$ for $\Gamma_{z}=0$ (blue, open diamonds) and $\Gamma_{z}=0.3 \Omega$ (black, open circles).

state for different $N$. For unitary system, $\Gamma_{r, z}=0$, we observe collective Rabi oscillations of $\left\langle n_{\mathrm{R}}\right\rangle$ between 0 and 1 with frequency $\sqrt{N} \Omega$. In the presence of a small Rydberg state decay, $\Gamma_{r} \neq 0$, these oscillations are slowly damped and the final steady state contains $\left\langle n_{\mathrm{R}}\right\rangle \simeq \frac{1}{2}$ Rydberg excitation, due to the saturation of the transition $|G\rangle \leftrightarrow\left|R^{(1)}\right\rangle$ between the collective ground state $|G\rangle=\left|g_{1}, g_{2}, \ldots, g_{N}\right\rangle$ and the symmetric single Rydberg excitation state $\left|R^{(1)}\right\rangle=\frac{1}{\sqrt{N}} \sum_{j}^{N}\left|g_{1}, g_{2}, \ldots, r_{j}, \ldots, g_{N}\right\rangle$ 22] $\left[\left\langle n_{\mathrm{R}}\right\rangle\right.$ slightly larger than $\frac{1}{2}$ seen in Fig. [1(b) is due to imperfect blockade of Rydberg excitation of the outermost atoms, $\left.\Delta\left(0.7 d_{\mathrm{b}}\right) \sim 10 w\right]$. Simultaneously, we have $Q \simeq-\frac{1}{2}$ consistent with the probabilities $p_{\mathrm{R}}(0) \simeq$ $p_{\mathrm{R}}(1) \simeq 0.5$.

Coherence relaxation with the rate $\Gamma_{z}$ comparable to $\Omega$ leads to $\left\langle n_{\mathrm{R}}\right\rangle$ approaching unity, as was also discussed in [22]. The reason for this is the population of all nonsymmetric single Rydberg excitation states of the system, which, upon saturation, leaves in the ground state $|G\rangle$ only a small fraction of population $p_{\mathrm{R}}(0) \simeq 1 /(N+1)$, with the result that $\left\langle n_{\mathrm{R}}\right\rangle \simeq p_{\mathrm{R}}(1) \simeq N /(N+1)$ 27]. From the simulations we obtain small, but not negligible, probabilities $p_{\mathrm{R}}(n) \ll 1$ of multiple Rydberg excitations $n \geq 2$, again due to imperfect blockade. For the mean number of Rydberg excitations $\left\langle n_{\mathrm{R}}\right\rangle \gtrsim 0.9$ for $N \geq 20$, we now have $Q \simeq-0.9$, Fig. 1(b).

Consider next the dynamics of the system in a larger volume. As the system size approaches the blockade distance, $d=d_{\mathrm{b}}$, the vdW interactions $\Delta(d) \simeq w$ between the atoms at the opposite sides of the confinement volume
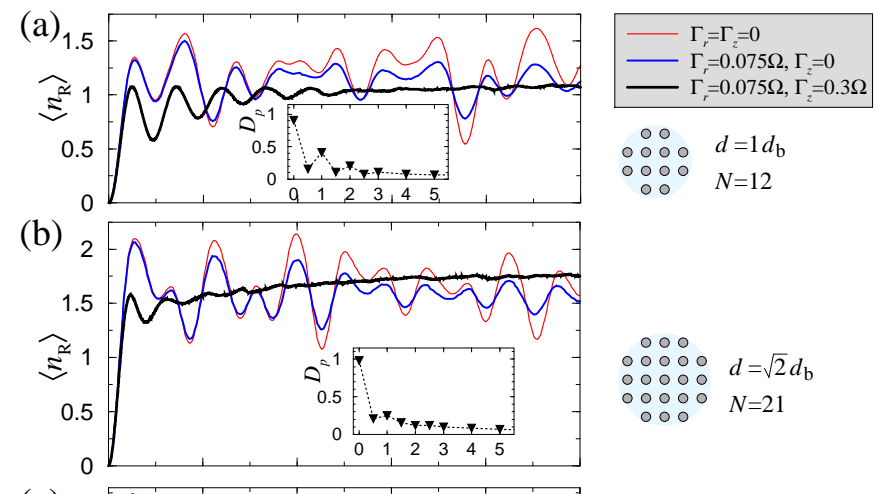

(c)

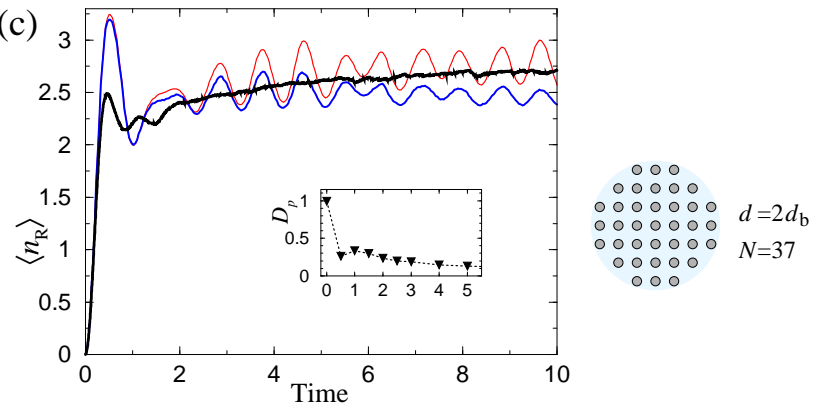

FIG. 2. Time-dependence of the number of Rydberg excitations $\left\langle n_{\mathrm{R}}\right\rangle$ of $N=12,21,37$ atoms in the volume of size $d=(1, \sqrt{2}, 2) d_{\mathrm{b}},(\mathrm{a}, \mathrm{b}, \mathrm{c})$, respectively. The inset in each graph shows the Kolmogorov distance $D_{p}$ between the probability distributions $p_{\{\sigma\}}(t)$ of configurations of Rydberg excitations of the system at different times $t$ and $p_{\{\sigma\}}(\infty)$ in the steadystate.

still suppress, but do not completely block, their Rydberg excitation, Fig. 2(a). In effect, these interactions dephase [19, 20] the periodic Rabi oscillations of $\left\langle n_{\mathrm{R}}\right\rangle$ even without the coherence relaxation, $\Gamma_{z}=0$. Remarkably, $\left\langle n_{\mathrm{R}}\right\rangle$ now fluctuates around 1 for both unitary and dissipative system. In the latter case, the coherence relaxation $\Gamma_{z}=0.3 \Omega$ quickly damps the dynamics and the system gradually approaches a steady state (see below). We observe similar behavior for larger systems, $d=\sqrt{2} d_{\mathrm{b}}$ and $d=2 d_{\mathrm{b}}$ in Fig. 2(b) and (c), which of course can accommodate more Rydberg excitations, $\left\langle n_{\mathrm{R}}\right\rangle \sim 1.5$ and $\left\langle n_{\mathrm{R}}\right\rangle \sim 2.5$, respectively.

In the long time limit, the dissipative system equilibrates. Figure 3 shows the steady-state values of $\left\langle n_{\mathrm{R}}\right\rangle$ and $Q$ versus the number of atoms $N$ in the confinement volume of diameter $d=(1, \sqrt{2}, 2) d_{\mathrm{b}}$. Clearly, larger volumes accommodate more Rydberg excitations, whose mean number $\left\langle n_{\mathrm{R}}\right\rangle$ is, however, not directly proportional to $\left(d / d_{\mathrm{b}}\right)^{2}$ as one might expect for a $2 \mathrm{D}$ system (and is probably true for very large volumes). This is a finitesize effect as the Rydberg excitations repel each other to the circular boundary of the confinement volume [17]. The non-smooth dependence of $\left\langle n_{\mathrm{R}}\right\rangle$ (and $Q$ ) on $N$ is also due to geometric constrains of arranging an integer number of atoms simultaneously in the square lattice and circle which results in different number of atoms closest to the boundaries of the volume. Note that similarly to 

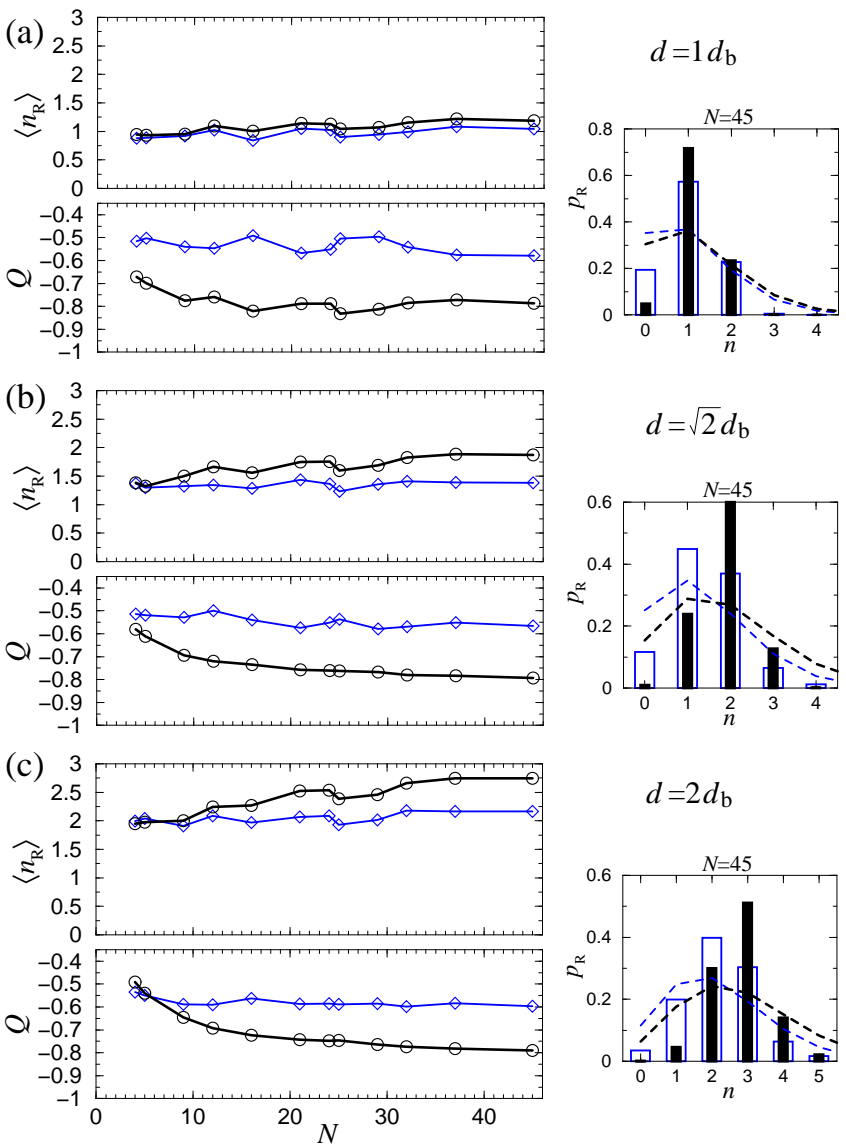

FIG. 3. Steady state values of the mean number of Rydberg excitations $\left\langle n_{\mathrm{R}}\right\rangle$ and the corresponding $Q$ parameter versus the number $N \leq 45$ of atoms in the volume of size $d=(1, \sqrt{2}, 2) d_{\mathrm{b}},(\mathrm{a}, \mathrm{b}, \mathrm{c})$, respectively, for $\Gamma_{r}=0.075 \Omega, \Gamma_{z}=0$ (blue lines, open diamonds) and $\Gamma_{z}=0.3 \Omega$ (black lines, open circles). The graphs on the right show the probability distributions $p_{\mathrm{R}}(n)$ for $N=45$ atoms in the corresponding volume: $\Gamma_{z}=0$ (blue open bars) and $\Gamma_{z}=0.3 \Omega$ (black filled bars). The dashed lines show the Poisson distribution for the corresponding $\left\langle n_{\mathrm{R}}\right\rangle$.

the small volume case, in the absence of coherence relaxation, $\Gamma_{z}=0$, the mean number of Rydberg excitations $\left\langle n_{\mathrm{R}}\right\rangle$ in a given volume changes little with $N$ while $Q$ stays close to $-\frac{1}{2}$. In contrast, for $\Gamma_{z} \lesssim \Omega,\left\langle n_{\mathrm{R}}\right\rangle$ slowly grows with the number of atoms $N$ (or density), while $Q \simeq-0.8$ indicates highly sup-Poissonian statistics of the number of Rydberg excitations, i.e. sharply peaked probability distribution $p_{\mathrm{R}}(n)$, Fig. 3 .

In the experiment, one prepares all the atoms in the ground state $|g\rangle$, applies the driving field $\Omega$ for a certain time $t$ and then performs projective measurements $\left\{\hat{\sigma}_{r r}^{j}\right\}$ of Rydberg excitations [17]. Every experimental sequence then results in a particular configuration $\left\{\hat{\sigma}_{r r}^{j}\right\} \rightarrow$ $\{0,0,1,0, \ldots\}$ of Rydberg excitations of the atoms. Averaging over many (typically several hundred) such experimental sequences yields the probabilities $p_{\{\sigma\}}(t)$ of various configurations, which are given by the diagonal elements of the density matrix $\hat{\rho}(t)$ of the system in the $\{|g\rangle,|r\rangle\}$ basis. To quantify the dynamical approach of the system to the steady-state, we calculate the Kolmogorov distance $D_{p}(t)=\frac{1}{2} \sum_{\{\sigma\}}\left|p_{\{\sigma\}}(t)-p_{\{\sigma\}}(\infty)\right|$, where $p_{\{\sigma\}}(\infty)$ are the steady-state probabilities, i.e. diagonal elements of $\hat{\rho}(\infty)$. The insets of Fig. 2 show $D_{p}(t)$ at different times $t$. For a few atoms within a small volume, we observe initial damped oscillations and rapid approach of the system to the steady state configuration of Rydberg excitations, Fig. 2(a). Increasing the number of atoms and the size of the system leads to less pronounced oscillations of $D_{p}(t)$ and slower equilibration, Figs. 2(b,c). This is to be expected since, on the one hand, more atoms at various distances from each other result in stronger dephasing, and, on the other hand, it takes now longer time to establish correlations between distant atoms which interact weakly, or negligibly, with each other. The similarly slow approach of a 1D lattice system to the global steady state was discussed in [28].

We note that the system attains its true steady-state on a time scale of $t \gtrsim 20 \mu \mathrm{s}$, which in an experiment can be prohibitively long due to the loss of atoms and the need of continuous laser irradiation. However, already after several $\mu$ s of laser driving [17], the probability distribution $p_{\{\sigma\}}$ of Rydberg excitation configurations is close to the steady-state distribution, with the distance between the two $D_{p} \lesssim 0.1$.

To conclude, we considered resonant Rydberg excitation of atoms in the presence of small population decay of the Rydberg states and sizable decay of atomic coherence, consistent with real experimental situations. We analyzed the dynamics of the many-body system and its approach to the steady state. An important and perhaps counterintuitive result that emerged from our studies is that the decay of atomic coherence, while destroying the quantum correlations, or entanglement, between the atoms, amplifies classical correlations, leading to narrower sub-Poissonian probability distribution of the number of Rydberg excitations quantified by larger negative values of the Mandel $Q$ parameter.

We finally note that for the parameters used in Fig. 3, the many-body steady state is not too far from the classical, for which the density matrix $\hat{\rho}_{\mathrm{cl}}(\infty)$ is diagonal in the $\{|g\rangle,|r\rangle\}$ basis. We have calculated the trace distance $D_{\rho}=\frac{1}{2} \operatorname{tr}\left|\hat{\rho}(\infty)-\hat{\rho}_{\mathrm{cl}}(\infty)\right|$ between the complete density matrix of the system $\hat{\rho}(\infty)$ and $\hat{\rho}_{\mathrm{cl}}(\infty)$, obtaining $D_{\rho} \simeq 0.2-0.3$. Increasing the relaxation rate $\Gamma_{z}$ decreases $D_{\rho}$, and for $\Gamma_{z} \simeq \Omega$ we obtain $D_{\rho} \lesssim 0.1$ and nearly constant $Q \simeq-0.85$ versus the atom number $N \gtrsim 20$ and $d>d_{\mathrm{b}}$. The near-diagonality of the density matrix $\hat{\rho}(\infty)$, i.e. smallness of inter-atomic coherences, suggest that the steady state of the many-body system can efficiently be simulated using semiclassical methods, such as, e.g., rate equations or Monte Carlo sampling [22]. The results of such simulations for large $2 \mathrm{D}$ systems will be reported elsewhere. 
[1] T.F. Gallagher, Rydberg Atoms (Cambridge University Press, Cambridge, 1994).

[2] M. Saffman, T.G. Walker, and K. Mølmer, Rev. Mod. Phys. 82, 2313 (2010).

[3] D. Comparat and P. Pillet, J. Opt. Soc. Am. B 27, A208 (2010).

[4] D. Tong, S.M. Farooqi, J. Stanojevic, S. Krishnan, Y.P. Zhang, R. Côté, E.E. Eyler, and P.L. Gould, Phys. Rev. Lett. 93, 063001 (2004).

[5] T. Vogt, M. Viteau, J. Zhao, A. Chotia, D. Comparat, and P. Pillet, Phys. Rev. Lett. 97, 083003 (2006).

[6] K. Singer, M. Reetz-Lamour, T. Amthor, L.G. Marcassa, and M. Weidemüller, Phys. Rev. Lett. 93, 163001 (2004).

[7] R. Heidemann, U. Raitzsch, V. Bendkowsky, B. Butscher, R. Löw, L. Santos, and T. Pfau, Phys. Rev. Lett. 99, 163601 (2007).

[8] R. Löw, H. Weimer, U. Krohn, R. Heidemann, V. Bendkowsky, B. Butscher, H.P. Büchler, and T. Pfau, Phys. Rev. A 80, 033422 (2009).

[9] M.D. Lukin, M. Fleischhauer, R. Côté, L.M. Duan, D. Jaksch, J.I. Cirac, and P. Zoller, Phys. Rev. Lett. 87, 037901 (2001).

[10] F. Robicheaux and J.V. Hernandez, Phys. Rev. A 72, 063403 (2005).

[11] J. Stanojevic and R. Côté, Phys. Rev. A 80, 033418 (2009).

[12] E. Urban, T.A. Johnson, T. Henage, L. Isenhower, D.D. Yavuz, T.G. Walker, and M. Saffman, Nature Phys. 5, 110 (2009); A. Gaëtan, Y. Miroshnychenko, T. Wilk, A. Chotia, M. Viteau, D. Comparat, P. Pillet, A. Browaeys, and P. Grangier, ibid 5, 115 (2009).

[13] Y.O. Dudin, L. Li, F. Bariani, and A. Kuzmich, Nature Phys. 8, 790 (2012).

[14] D. Petrosyan and K. Mølmer, Phys. Rev. A 87, 033416 (2013).
[15] T. Cubel Liebisch, A. Reinhard, P.R. Berman, and G. Raithel, Phys. Rev. Lett. 95, 253002 (2005).

[16] M. Viteau, P. Huillery, M.G. Bason, N. Malossi, D. Ciampini, O. Morsch, E. Arimondo, D. Comparat, and P. Pillet, Phys. Rev. Lett. 109, 053002 (2012).

[17] P. Schauß, M. Cheneau, M. Endres, T. Fukuhara, S. Hild, A. Omran, T. Pohl, C. Gross, S. Kuhr, and I. Bloch, Nature 491, 87 (2012).

[18] A. Schwarzkopf, R.E. Sapiro, and G. Raithel, Phys. Rev. Lett. 107, 103001 (2011).

[19] T.A. Johnson, E. Urban, T. Henage, L. Isenhower, D.D. Yavuz, T.G. Walker, and M. Saffman, Phys. Rev. Lett. 100, 113003 (2008).

[20] U. Raitzsch, V. Bendkowsky, R. Heidemann, B. Butscher, R. Löw, and T. Pfau, Phys. Rev. Lett. 100, 013002 (2008).

[21] M. Gärttner, K.P. Heeg, T. Gasenzer and J. Evers, Phys. Rev. A 86, 033422 (2012); arXiv:1203.2884.

[22] D. Petrosyan, M. Höning, and M. Fleischhauer, arXiv:1212.2423

[23] P. Lambropoulos and D. Petrosyan, Fundamentals of Quantum Optics and Quantum Information, (Springer, Berlin, 2007).

[24] K. Singer, J. Stanojevic, M. Weidemüller, and R. Côté, J. Phys. B 38, S295 (2005).

[25] J. Dalibard, and Y. Castin, and K. Mølmer Phys. Rev. Lett. 68, 580 (1992); R. Dum, P. Zoller, and H. Ritsch, Phys. Rev. A 45, 4879 (1992); C.W. Gardiner, A.S. Parkins, and P. Zoller Phys. Rev. A 46, 4363 (1992); M.B. Plenio and P.L. Knight, Rev. Mod. Phys. 70, 101 (1998).

[26] L. Mandel, Opt. Lett. 4, 205 (1979).

[27] J. Honer, R. Löw, H. Weimer, T. Pfau, and H.P. Büchler, Phys. Rev. Lett. 107, 093601 (2011).

[28] M. Höning, D. Muth, D. Petrosyan, and M. Fleischhauer, Phys. Rev. A 87, 023401 (2013). 